\title{
SPARC and Vav3 Expression in Meningioma: Factors Related to Prognosis
}

\author{
Jie Jiang, Yuejia Song, Nan Liu, Chengjun Lin, Shiguang Zhao, Yu Sun, Zhen \\ Zhang, Xiang Fang, Jiping Qi
}

\begin{abstract}
Background: Meningiomas account for approximately 24-30\% of primary intracranial neoplasms. Histopathologic grade and degree of resection are two major prognostic factors. The aim of this study was to determine the factors associated with the prognosis of meningioma. Methods: We used immunohistochemistry to analyze the expression levels of Vav3, SPARC, p-Akt, cyclin D1, and Ki-67 in 287 meningiomas of all grades. Results: The expression of Vav3, SPARC, p-Akt, cyclin D1, and Ki67 significantly increased with meningioma grade $(\mathrm{p}<0.01)$, and was higher in brain-invasive meningiomas compared to non-invasive meningiomas (WHO grade I) $(p<0.05)$. Furthermore, the expression of Vav3, p-Akt, and Ki-67 was higher in recurrent meningiomas compared to non-recurrent meningiomas (WHO grade I) (p<0.05). Conclusion: The expression of Vav3, SPARC, p-Akt, cyclin D1, and Ki-67 in meningiomas appears to correlate with meningioma invasiveness, aggressiveness, and recurrence.
\end{abstract}

\begin{abstract}
RÉSUMÉ: Expression de SPARC et de Vav3 dans le méningiome : facteurs reliés au pronostic. Contexte : les méningiomes constituent à peu près 24 à $30 \%$ des néoplasies intracrâniennes primitives. Le grade histopathologique et le degré de résection de la tumeur sont deux facteurs de pronostic importants. Le but de cette étude était de déterminer les facteurs associés au pronostic du méningiome. Méthode : nous avons utilisé l'immunohistochimie pour analyser le niveau d'expression de Vav3, SPARC, p-AKt, cycline D1 et Ki-67 dans 287 méningiomes de tout grade. Résultats : L'expression de Vav3, SPARC, p-Akt, cycline D1 et Ki-67 augmentait significativement avec l'augmentation du grade de la tumeur $(\mathrm{p}<0,01)$ et elle était plus élevée dans les méningiomes envahissants que dans les méningiomes non envahissants (OMS grade 1) $(\mathrm{p}<0,05)$. De plus, l'expression de Vav3, p-Akt et Ki-67 était plus élevée dans les méningiomes récurrents que dans les méningiomes non récurrents (Grade I de l'OMS) (p < 0,05). Conclusion : L'expression de Vav3, SPARC, p-Akt, cycline D1 et Ki-67 dans les méningiomes semble corrélée au caractère invasif et agressif du méningiome ainsi qu'à sa récurrence.
\end{abstract}

Can J Neurol Sci. 2013; 40: 814-818

Meningiomas account for approximately 24-30\% of primary intracranial neoplasms. They arise from arachnoidal cap cells, and are associated with cytogenetic alterations involving deletion of chromosome $22^{1}$. Meningiomas usually occur in aged women, with a female to male ratio of $1.7: 1$, possibly due to sex hormones ${ }^{2}$. According to the 2007 World Health Organization (WHO) classification system, meningiomas can be divided into WHO grades I, II, and III by their histopathological appearance. Brain-invasive meningiomas are prognostically considered WHO grade $\mathrm{II}^{3}$. The prognosis for meningiomas is commonly dependent upon histopathologic grade and degree of resection. Although the majority of meningiomas are classified as WHO grade I, recurrence can occur despite complete excision of the tumor. There are few known prognostic biomarkers for meningiomas. Therefore, the goal of our study was to investigate the expression levels of secreted protein acidic and rich in cysteine (SPARC), the guanine nucleotide exchange factor Vav3, phosphorylated Akt (p-Akt), cyclin D1, and Ki-67, a cellular marker for proliferation, in all meningiomas, and determine its correlation with invasiveness, recurrence, and progression.

From the Department of Pathology (JJ, NL, YSu, ZZ, XF, JQ), Department of Digestive System (YSo), Department of Neurosurgery (SZ), First Affiliated Hospital of Harbin Medical University; Department of Anesthesiology (CL), Fourth Affiliated Hospital of Harbin Medical University, Harbin, PR China.

Received November 9, 2012. Final Revisions Submitted July 8, 2013. Correspondence to: Jiping Qi, Department of Pathology, First Affiliated Hospital of Harbin Medical University, 23 Youzheng Street, Nangang District, Harbin 150001, PR China.Email: bless88775469@163.com. 


\section{Patients And Methods}

\section{Patients}

Specimens were collected from patients diagnosed with meningiomas who were operated on at The Neurosurgical Department of the First Affiliated Hospital of Harbin Medical University (China) between 2003 and 2009. All patients gave informed consent prior to their participation in the study. All WHO grade II (139) and grade III (13) cases, but only 135 grade I cases (diagnosed from 2003 to 2004 with non-recurrent, noninvasive, completely resected meningioma), and an additional 27 recurrent cases (WHO grade I) were collected. Only the tongues of meningioma cohesive cells infiltrating the cortex with benign histopathology were classified as brain-invasive. The few observed cases of secretory, lymphoplasmacyte-rich, metaplastic meningiomas were classified as 'mixed'.

\section{Immunohistochemistry}

Paraffin sections ( $4 \mathrm{~mm}$ ) were mounted onto glass slides. The slides were deparaffanized at $60^{\circ} \mathrm{C}$ for one hour (h), and then dewaxed in xylene. Antigen retrieval was achieved by immersing the slides in citrate buffer or EDTA in an autoclave for 2 or 2.5 minutes (min). The samples were incubated with primary antibody for $20 \mathrm{~min}$ in a water bath at $37^{\circ} \mathrm{C}$, followed by incubation with secondary antibody for $20 \mathrm{~min}$. The following primary antibodies were used: 1:300 dilution of Vav3 (ab21208, goat polyclonal, abcam; Cambridge, UK), 1:100 dilution of SPARC (ab89739, abcam; mouse monoclonal), 1:50 dilution of p-Akt (4060s, rabbit polyclonal; Cell Signaling Technology, Beverly, MA, USA), 1:30 dilution of cyclin D1 (ERR2241, rabbit monoclonal; Biogenex, Fremont, CA, USA), and a 1:100 dilution of Ki-67 (BGX-Ki-67, mouse monoclonal; Biogenex, Fremont, CA, USA). Antibodies were visualized with 3,3diaminobenzidine (DAB). Control slides were prepared by following the same procedures without applying primary antibody.

\section{Evaluation of immunostaining}

Immunohistochemical staining was independently evaluated by two neuropathologists who were blinded to WHO grade and recurrence status. The interobserver variation and intraobserver reliability of the selected factors did not have statistical significance $(\mathrm{p}>0.05)$. Positive SPARC cytoplasmic staining was observed. Staining was scored as: 0 (no staining), +1 (weak staining; $<20 \%$ ), +2 to +3 (moderate staining; $20-50 \%$ ), or +4 to +5 (strong staining; $>50 \%$ ). A numerical value from 0 to 3 was used for further analysis ${ }^{4}$. The percentage of cells expressing $\mathrm{Ki}$ 67 and cyclin D1 were obtained from cells with the highest staining intensity in 10 high-power fields (HPF). The percentage of labeled cells $=$ number of labeled cells/total counted cells $\times 100^{5}$. The intensity and extent of positive Vav3 cytoplasmic labeling were semi-quantitatively assessed and scored as 0 (no staining), +1 (weak and focal staining in $<25 \%$ of tissue),+2 (moderate intensity in 25-50\% of tissue), +3 (moderate intensity in $>50 \%$ of tissue), and +4 (strong and diffuse staining in $>50 \%$ of tissue ${ }^{6}$. Immunostaining for $\mathrm{p}$-Akt showed cytoplasmic expression, and was designated $0,+1,+2$, and +3 , which represented no cell staining, 20\% cell staining (weak), 20-50\% cell staining (moderate), and $>50 \%$ cell staining (strong), respectively ${ }^{7}$.

\section{Statistical analysis}

All analyses were carried out with SPSS statistical software, version 19.0. Descriptive statistical methods of mean, frequency, and standard deviation were applied. Quantitative variables ( $\mathrm{F}$ and $\mathrm{T}$ test) or qualitative variables (chi-squared tests) were used to determine the significance of difference between groups. Spearman's rank correlation coefficient was used to determine the relationship between factors.

\section{RESULTS}

\section{Clinicopathological data}

A total of 287 patients (not including the 27 recurrent cases) were included in our study. Most patients $(84 \%)$ were at least 40

\begin{tabular}{|c|c|c|c|}
\hline & grade & cases & mean \pm SD \\
\hline \multirow[t]{5}{*}{$\mathrm{Ki}-67$} & I & 135 & $0.87 \pm 0.12$ \\
\hline & II & 139 & $4.27 \pm 0.45^{\mathrm{a}}$ \\
\hline & III & 13 & $13.00 \pm 0.73^{\mathrm{a}}$ \\
\hline & recurrence & 27 & $3.44 \pm 0.37^{\mathrm{a}}$ \\
\hline & Brain invasive & 56 & $3.00 \pm 0.02^{\mathrm{a}}$ \\
\hline \multirow[t]{5}{*}{ Cyclin D1 } & I & 135 & $32.37 \pm 1.74$ \\
\hline & II & 139 & $47.16 \pm 1.81^{\mathrm{b}}$ \\
\hline & III & 13 & $54.62 \pm 2.61^{\mathrm{b}}$ \\
\hline & recurrent & 27 & $18.74 \pm 2.03^{b}$ \\
\hline & Brain invasive & 56 & $45.09 \pm 1.98^{\mathrm{b}}$ \\
\hline
\end{tabular}

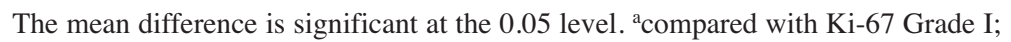

bcompared with Cyclin D1 Grade I 


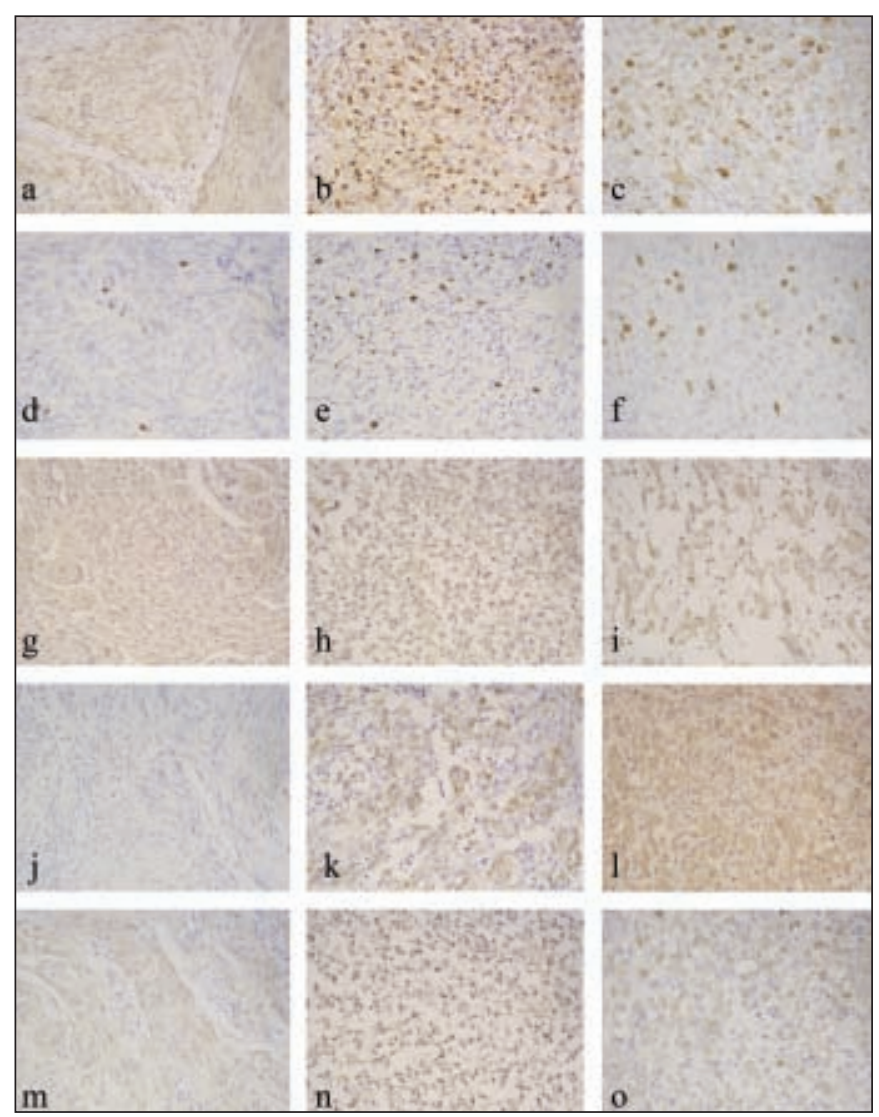

Figure: The expression of factors in all grade meningiomas $(\times 40)$ a) Cyclin D1 grade I, b) Cyclin D1 grade II, c) Cyclin D1 grade III, d) Ki67 grade I, e) Ki67 grade II, f) Ki67 grade III, g) SPARC grade I, h) SPARC grade II, i) SPARC grade III, j) p-Akt grade I, $k$ ) p-Akt grade II, l) p-Akt grade III, $m$ ) Vav3 grade I, $n$ ) Vav3 grade II, o) Vav3 grade III.

years of age. Half of the females $(50.2 \%)$ had grade I meningiomas, and $59.0 \%$ of the male cases were grade II meningiomas. Meningiomas located over the convexity of the brain comprised $60.6 \%$ of all cases. Brain-invasive (40.3\%), atypical $(32.4 \%)$, chordoid $(4.3 \%)$, and clear cell $(23.0 \%)$ meningiomas were classified as WHO grade II.

\section{Ki-67 and cyclin D1 expression in meningiomas}

Positive nuclear staining of Ki-67 was observed in the meningiomas, and many cases showed patterns of diffuse cytoplasmic and nuclear cyclin D1 staining (Figure). The expression levels of Ki-67 and cyclin D1 were significantly higher in grade II and grade III meningiomas compared to grade I meningiomas $(\mathrm{p}<0.01$; Table 1$)$. Furthermore, there was increased Ki-67 and cyclin D1 expression in brain-invasive meningiomas compared to non-invasive meningiomas (WHO grade I) $(\mathrm{p}<0.05)$. The expression of Ki-67 was significantly higher in recurrent meningiomas compared to non-recurrent meningiomas (WHO grade I) $(\mathrm{p}<0.01)$. There was a statistically significant positive correlation between Ki-67 and cyclin D1 expression according to Spearman's rank-order correlation (Table 2).

\section{Expression of SPARC and p-Akt in meningiomas}

The expression of SPARC was significantly higher in grade II and III meningiomas compared to grade I meningiomas $(\mathrm{p}<0.01)$ (Table 3). A statistically significant difference in p-Akt expression was detected in grade II and III meningiomas $(\mathrm{p}<0.01)$ (Table 3). Although positive cytoplasmic expression of p-Akt was found in a few grade I meningiomas, most grade II and III meningiomas had both cytoplasmic and nuclear p-Akt expression (Figure). There was a statistically significant positive correlation between Ki-67 and SPARC expression, and between SPARC and p-Akt expression according to Spearman's rankorder correlation (Table 2). In addition, SPARC and p-Akt expression was higher in brain-invasive meningiomas compared to grade I meningiomas $(\mathrm{p}<0.01)$ (Table 3 ). Interestingly, there was higher p-Akt expression in the tumor-brain border of three brain-invasive meningiomas compared to the tumor itself. In addition, p-Akt expression was higher in recurrent meningiomas compared to grade I meningiomas $(\mathrm{p}<0.05)$.

\section{Vav3 expression in meningiomas}

The expression levels of Vav3 were significantly higher in grade II and III meningiomas compared to grade I meningiomas $(\mathrm{p}<0.01$; Table 3$)$. A positive correlation was found between $\mathrm{Ki}$ 67 and Vav3 expression, and between Vav3 and p-Akt

Table 2: Spearman's analysis of the correlation between immunoexpression of factors in all grade meningiomas

\begin{tabular}{llllllll}
\hline & & Cyclin D1 & Ki-67 & SPARC & p-Akt & Vav3 & grade \\
\hline Cyclin D1 & Spearman r & 1.000 & $0.356^{* *}$ & $0.162^{*}$ & $0.298^{* *}$ & $0.162^{* *}$ & $.422^{* *}$ \\
& Sig.(2-tailed) &. & 0.000 & 0.006 & 0.000 & 0.006 & 0.000 \\
Ki-67 & Spearman r & $0.356^{* *}$ & 1.000 & $0.203^{* *}$ & $0.397^{* *}$ & $0.228^{* *}$ & $0.590^{* *}$ \\
& Sig.(2-tailed) & 0.000 &. & 0.001 & 0.000 & 0.000 & 0.000 \\
SPARC & Spearman r & $0.162^{* *}$ & $0.203^{* *}$ & 1.000 & $0.314^{* *}$ & $0.242^{* *}$ & $0.369^{* *}$ \\
& Sig.(2-tailed) & 0.006 & 0.001 &. & 0.000 & 0.000 & 0.000 \\
p-Akt & Spearman r & $0.298^{* *}$ & $0.397^{* *}$ & $0.314^{* *}$ & 1.000 & $0.386^{* *}$ & $0.701^{* *}$ \\
& Sig.(2-tailed) & 0.000 & 0.000 & 0.000 &. & 0.000 & 0.000 \\
Vav3 & Spearman r & $0.162^{* *}$ & $0.228^{* *}$ & $0.242^{* *}$ & $0.386^{* *}$ & 1.000 & $0.358^{* *}$ \\
& Sig.(2-tailed) & 0.006 & 0.000 & 0.000 & 0.000 &. & 0.000 \\
grade & Spearman r & $.422^{* *}$ & $0.590^{* *}$ & $0.369^{* *}$ & $0.701^{* *}$ & $0.358^{* *}$ & 1.000 \\
& Sig.(2-tailed) & 0.000 & 0.000 & 0.000 & 0.0000. & 0.000 &. \\
\hline
\end{tabular}

**In the credibility of 0.01 (two-tailed), the correlation is significant. *In the credibility of 0.05 (two-tailed), the correlation is significant. 
Table 3: Comparison of SPARC, Vav3 and p-Akt immunohistochemical expression in all grade meningiomas

\begin{tabular}{clcccccc}
\hline Factor & Group & 0 & 1 & 2 & 3 & total & P \\
\hline SPARC & Grade I & 50 & 0 & 61 & 24 & 135 & \\
& Grade II & 1 & 1 & 88 & 49 & 139 & $0.000^{\mathrm{a}}$ \\
& Grade III & 0 & 0 & 10 & 3 & 13 & $0.000^{\mathrm{a}}$ \\
& brain-invasive & 49 & 3 & 30 & & 56 & $0.000^{\mathrm{a}}$ \\
& recurrent & 139 & 13 & 56 & & 27 & $0.064^{\circ}$ \\
Vav3 & Grade I & 6 & 119 & 10 & 0 & 135 & \\
& Grade II & 4 & 103 & 29 & 3 & 139 & $0.002^{\mathrm{b}}$ \\
& Grade III & 0 & 0 & 5 & 8 & 13 & $0.000^{\mathrm{b}}$ \\
& brain-invasive & 0 & 0 & 42 & 14 & 56 & $0.001^{\mathrm{b}}$ \\
& recurrent & 2 & 16 & 5 & 4 & 27 & $0.008^{\mathrm{b}}$ \\
& Grade I & 117 & 14 & 4 & 0 & 135 & \\
& Grade II & 28 & 54 & 47 & 9 & 139 & $0.000^{\mathrm{c}}$ \\
& Grade III & 0 & 2 & 2 & 9 & 13 & $0.000^{\mathrm{c}}$ \\
& brain-invasive & 0 & 0 & 42 & 14 & 56 & $0.000^{\mathrm{c}}$ \\
& recurrent & 15 & 7 & 3 & 1 & 26 & $0.000^{\mathrm{c}}$ \\
\hline
\end{tabular}

The mean difference is significant at the 0.05 level. ${ }^{\text {a }}$ compared with SPARC grade I;

bcompared with Vav3 grade I; ${ }^{c}$ compared with SPARC grade I

expression according to Spearman's rank-order correlation (Table 2). Expression of Vav3 was significantly higher in braininvasive and recurrent meningiomas compared to grade I meningiomas $(\mathrm{p}<0.05)$.

\section{Discussion}

Extensive research has been carried out to identify markers that predict meningioma prognosis; however, there remains a lack of reliable biological prognostic markers. This study was carried out to investigate Vav3, SPARC, p-Akt, cyclin D1, and $\mathrm{Ki}-67$ expression in a relatively large number of meningioma cases, particularly in grade II meningiomas. Few studies have shown significant changes in SPARC, p-Akt, and cyclin D1 expression in meningiomas, and to date, no studies have investigated Vav3 expression in meningiomas.

The Ki-67/MIB-1 proliferation index is related to the grade of meningioma; Ki-67 expression is the only factor that can independently indicate both recurrence and overall survival ${ }^{8}$. Cyclin D1 is an essential nuclear protein, that allows cells to move through the G1 phase of the cell cycle. The expression of cyclin D1 and proliferating cell nuclear antigen is increased in grade II and III meningiomas ${ }^{9}$. Our findings are consistent with this study, as we found an increase in cyclin D1 expression in brain-invasive meningiomas compared to grade I meningiomas. These data indicate that cyclin D1 is associated with tumor progression and invasiveness. In addition, we found diffuse cytoplasmic and nuclear cyclin D1 staining, although the significance of these different expression patterns is unclear. The expression of Cyclin D1 in combination with Ki-67 expression, might be related to the proliferation, invasion, and progression of meningiomas.

The expression of SPARC increases in some tumors, and decreases in others ${ }^{10}$. Matrix-associated staining of SPARC in our study was consistent with the features of SPARC as a secreted protein. SPARC was originally thought to be a potential marker of brain-invasive meningiomas ${ }^{4}$; however, this finding has been controversial. Positive cells were found in both invasive and non-invasive tumors, and disruption of the pialglial basement membrane was associated with grade of meningioma malignancy ${ }^{11}$. Higher SPARC expression was detected in atypical and anaplastic meningiomas, as well as in recurrent meningiomas ${ }^{12}$. There was higher SPARC and p-Akt expression in grade II and III meningiomas compared to grade I meningiomas in our study. The expression of SPARC and p-Akt was significantly higher in brain-invasive meningiomas, and pAkt expression was significantly higher in recurrent meningiomas. The data from our study suggest that SPARC and p-Akt expression might be associated with tumor invasion, progression, and recurrence.

The main signaling pathway in meningioma is the PI3K-Akt pathway. Previous studies have demonstrated increased p-Akt expression in atypical and malignant meningiomas compared to benign meningiomas, and the PI3K inhibitor, wortmannin, was able to inhibit tumor cell proliferation ${ }^{13}$. Our study showed a positive correlation between SPARC and p-Akt expression according to Spearman's rank-order correlation, suggesting that SPARC might function through the PI3K-Akt pathway in meningiomas. Cytoplasmic staining of p-Akt was observed in grade I meningiomas, and mostly nuclear p-Akt immunoreactivity in grade II and III meningiomas, combined with a poor disease prognosis. Higher p-Akt expression in grade II and III meningiomas indicate that the PI3K/Akt signaling pathway might be a potential target for meningioma therapy. The role of SPARC in some tumors might be associated with alterations in the methylation state of the SPARC promoter; thus, targeting SPARC may be an effective tumor therapy ${ }^{14}$.

Vav3 is a guanine nucleotide exchange factor for Rho family GTPases. Overexpression of Vav3 has been found in human prostate cancer, and may activate the androgen receptor axis through the PI3K/Akt pathway to promote cell growth ${ }^{6}$. Lee et al found that majority of breast cancers overexpress Vav3, which can stimulate breast cancer cell growth through the PI3K/Akt pathway ${ }^{15}$. In our study, Vav3 was expressed in meningiomas, and its expression increased with meningioma grade. In addition, 
there was a positive correlation between the expression of Vav3 and p-Akt according to Spearman's rank-order correlation. This suggests that Vav3 might function through the PI3K-Akt pathway in meningiomas. Vav3 expression was significantly higher in brain-invasive meningiomas, and also increased in recurrent cases, suggesting that Vav3 might be related to histopathology grade, invasiveness, and recurrence of meningioma.

According to the WHO system, brain-invasive meningiomas should prognostically be considered WHO grade II. In our study, the expression of SPARC, p-Akt, Vav3, Ki-67, and cyclin D1 were significantly higher in brain-invasive meningiomas compared to non-invasive meningiomas, and brain-invasive meningiomas appeared to have a poor prognosis. In grade II meningiomas, Ki-67 expression was higher in the atypical subtype compared to the brain-invasive and clear cell subtypes, and SPARC expression was higher in the brain-invasive subtype compared to atypical and clear cell subtypes(data not shown).

By examining a large number of meningioma cases, we found that the expression of Vav3, SPARC, p-Akt, cyclin D1, and Ki67 might be associated with meningioma progression, invasiveness, and recurrence. In addition, our data suggest that Vav3 and SPARC might function through the PI3/Akt pathway in meningiomas.

\section{REFERENCES}

1. Claus EB, Bondy ML, Schidkraut JM, Wiemels JL, Wrensch M, Black PM. Epidemiology of intracranial meningioma. Neurosurgery. 2005;57:1088-95.

2. Cordera S, Bottacchi E, D'Alessandro G, Machado D, De Gonda F, Corso G. Epidemiology of primary intracranial tumors in NW Italy, a population based study: stable incidence in the last two decades. J Neurol. 2002;249:281-4.

3. Perry A, Louis DN, Scheithauer BW, Budka H, von Deimling A. Meningiomas. In: Louis DN, Ohgaki H, Wiestler OD, Cavanee W.K, editors. World Health Organization Classification of Tumours of the Central Nervous System . Lyon: IARC Press; 2007. p. 164-72.

4. Rempel SA, Ge S, Gutiérrez JA. SPARC: A potential diagnostic marker of invasive meningiomas. Clin Cancer Res. 1999;5: 237-41.

5. Milenkovic S, Marinkovic T, Jovanovic MB, Djuricic S, Berisavac II, Berisavac I. Cyclin D1 immunoreactivity in meningiomas. Cell Mol Neurobiol. 2008;28:907-13.

6. Dong ZY, Liu Y, Lu S, et al. Vav3 oncogene is overexpressed and regulates cell growth and androgen receptor activity in human prostate cancer. Mol Endocrinol. 2006;20(10):2315-25.

7. Malik SN, Brattain M, Ghosh PM, et al. Immunohistochemical demonstration of Phospho-Akt in High Gleason Grade Prostate Cancer. Clin Cancer Res. 2002;8:1168-71.

8. Bruna J, Brell M, Ferrer I, Gimenez-Bonafe P, Tortosa A. Ki-67 proliferative index predicts clinical outcome in patients with atypical or anaplastic meningioma. Neuropathology. 2007;27: 114-20.

9. Alama A, Barbieri F, Spaziante R, et al. Significance of cyclin D1 expression in meningiomas: A preliminary study. J Clin Neurosci. 2007; $14: 355-58$.

10. Wong S, Crowley D, Bronson R, Hynes R. Analyses of the role of endogenous SPARC in mouse models of prostate and breast cancer. Clin Exp Metast. 2008;25:109-18.

11. Schittenhelm J, Mittelbronn M, Roser F, Tatagiba M, Mawrin C, Bornemann A. Patterns of SPARC expression and basement membrane intactness at the tumour-brain border of invasive meningiomas. Neuropath Appl Neuro. 2006;32: 525-31.

12. Bozkurt SU, Ayan E, Bolukbasi F, Elmaci I, Pamir N, Sav A. Immunohistochemical expression of SPARC is correlated with recurrence, survival and malignant potential in meningiomas. APMIS. 2009; 117:651-59.

13. Seno T, Harada H, Kohno S, Teraoka M, Inoue A, Ohnishi T. Downregulation of SPARC expression inhibits cell migration and invasion in malignant gliomas. Int J Oncol. 2009; 34:707-15.

14. Mawrin C, Sasse T, Kirches E, et al. Different activation of mitogen-activated protein kinase and akt signaling is associated with aggressive phenotype of Human meningiomas. Clin Cancer Res. 2005;11(11):4074-82.

15. Lee K, Liu Y, Mo JQ, Zhang J, Dong ZY, Lu S. Vav3 oncogene activates estrogen receptor and its overexpression may be involved in human breast cancer. BMC Cancer. 2008;8:158. 